\title{
POTENCIAL VULNERABILIDAD FRENTE A INUNDACIONES DE LOS ESTABLECIMIENTOS DE SALUD PÚBLICOS DE CUATRO REGIONES DEL NORTE DEL PERÚ
}

\author{
Akram Hernández-Vásquez ${ }^{1, a}$, Hugo Arroyo-Hernández ${ }^{2, b}$, Guido Bendezú-Quispe $^{3, c}$, Deysi Díaz-Seijas $^{4, d}$, \\ Stalin Vilcarromero ${ }^{5, \mathrm{e}}$, Juan Rubilar-González ${ }^{6, \mathrm{f}}$, Edith Gutierrez-Lagos ${ }^{7, \mathrm{~g}}$
}

\begin{abstract}
RESUMEN
Con el objetivo de determinar la potencial vulnerabilidad de los establecimientos de salud públicos de cuatro regiones del norte del Perú ante los posibles efectos del fenómeno El Niño (ENSO), se realizó un análisis espacial exploratorio con los puntos georreferenciados de las zonas de riesgos por activación de quebradas, reportadas por la Autoridad Nacional del Agua, y los establecimientos de salud públicos del Ministerio de Salud. Se simularon áreas de influencia concéntricas desde los puntos de riesgo hacia los establecimientos de salud públicos en un radio de 200, 1000 y 1500 metros. La región Tumbes sería la más afectada con el $37,2 \%$ de sus establecimientos afectados por inundaciones o deslizamientos. Las categorías I-2 y I-3 serían las más afectadas con el 28,9 y 31,6\% respectivamente. En conclusión, se identificaron establecimientos de salud cercanos a puntos de riesgo que podrían ser afectados ante la presencia del ENSO.
\end{abstract}

Palabras clave: Sistemas de información geográfica; Análisis espacial; Fenómeno El Niño; Establecimientos de salud; Perú (fuente: DeCS BIREME).

\section{POTENTIAL VULNERABILITY TO FLOODING AT PUBLIC HEALTH FACILITIES IN FOUR NORTHERN REGIONS OF PERU}

\begin{abstract}
In order to determine the potential vulnerability of public health facilities in four northern regions of Peru to the possible effects of EI Niño-Southern Oscillation (ENSO) phenomenon. An exploratory spatial analysis was performed using the geo-referenced points for at-risk areas based on the activation of gullies that were reported by the National Water Authority, and the location of the four regional public health facilities of the Ministry of Health. Concentric areas of influence were simulate from the points of risk towards the public health facilities using radii of 200, 1000 and 1500 meters. The Tumbes region would be the most affected with $37.2 \%$ of its health facilities being affected by floods and landslides. The I-2 and I-3 categories of health facilities appeared to be the most affected with $28.9 \%$ and $31.6 \%$ respectively. Therefore, public health facilities near the risk zones may be affected by the ENSO.
\end{abstract}

Key words: Geographic information systems; Spatial analysis; El Niño-Southern Oscillation; Health facilities; Peru (source: MeSH NLM).

\section{INTRODUCCIÓN}

El Perú es un país que constantemente se ve enfrentado a desastres naturales que conllevan al desplazamiento y hacinamiento de personas, favorecen la aparición y reemergencia de enfermedades, y la pérdida de capacidad en el cumplimiento de estrategias de control sanitario ${ }^{(1-3)}$. El sector salud tiene un rol fundamental

\footnotetext{
Universidad de Buenos Aires. Buenos Aires, Argentina.

Oficina General de Información y Sistemas. Instituto Nacional de Salud. Lima, Perú.

Facultad de Medicina, Universidad Nacional San Luis Gonzaga. Ica, Perú.

Instituto Nacional Cardiovascular, EsSalud. Lima, Perú.

Sociedad Científica de Estudiantes de Medicina de la Amazonía Peruana. Facultad de Medicina, Universidad Nacional de la Amazonía Peruana. Iquitos, Perú. Universidad Mayor. Temuco, Chile.

Ministerio de Energía. Santiago de Chile, Chile.

a Médico cirujano, magíster en Gestión y Políticas Públicas; ${ }^{\text {b }}$ médico cirujano; ${ }^{\mathrm{c}}$ estudiante de Medicina; ${ }^{\mathrm{d}}$ enfermera, especialista en Cuidados Intensivos; ${ }^{\mathrm{e}}$ médico cirujano, magíster en Salud Pública; ${ }^{\mathrm{f}}$ sociólogo; ${ }^{\mathrm{g}}$ geógrafa.
}

Parte de los resultados del estudio fueron presentados en la modalidad de póster en el IX Congreso Internacional del Instituto Nacional de Salud, 11 al 13 de noviembre de 2015, Lima, Perú.

Recibido: 19-01-16 Aprobado: 09-03-16

Citar como: Hernández-Vásquez A, Arroyo-Hernández H, Bendezú-Quispe G, Díaz-Seijas D, Vilcarromero S, Rubilar-González J, Gutierrez-Lagos E. Potencial vulnerabilidad frente a inundaciones, de los establecimientos de salud públicos de cuatro regiones del norte del Perú. Rev Peru Med Exp Salud Publica. 2016;33(1):92-9. doi: 10.17843/rpmesp.2016.331.2012 
antes, durante y después de la ocurrencia de estos fenómenos, para lo cual, es necesario garantizar el acceso a los servicios de salud a la población afectada.

El fenómeno El Niño o ENSO (del inglés El Niño Southern Oscillation), es un fenómeno climático océanoatmósfera a gran escala, vinculado con un calentamiento periódico de las temperaturas de la superficie marina en todo el ecuatorial central y centro-este del Pacífico, que se manifiesta aproximadamente cada cinco años, entre los meses de diciembre a marzo y tiene como consecuencias el incremento de la temperatura atmosférica; el incremento de las precipitaciones y de la descarga de los ríos y, dependiendo de la intensidad, lluvias torrenciales, inundaciones y deslizamientos de tierra ${ }^{(1,4)}$. En algunas regiones del Perú, como la costa norte, ha producido desastres naturales debido a las lluvias extremas e inundaciones ${ }^{(5)}$, que también afectaron seriamente la infraestructura de los establecimientos de salud (EE. SS.) tal como ocurrió con el ENSO de 1997-1998 en el que 557 EE. SS. fueron destruidos o presentaron daños en sus estructuras o equipos, lo cual no permitió una adecuada respuesta sanitaria ${ }^{(6)}$.

Ante una situación de desastre por lluvias e inundaciones, los servicios de salud pueden afectar su funcionamiento de diversas formas, incluyendo: bloqueo o destrucción de rutas de acceso a los establecimientos; daño a las estructuras; interrupción de la cadena de suministros a los EE. SS. (fármacos y material médico); fallo en el sistema de referencia y contrarreferencia; incremento de la demanda de atención, entre otros, que conducen a la sobrecarga de los EE. SS. y el fallo en su capacidad resolutiva ${ }^{(7)}$.

El Comité Multisectorial encargado del Estudio Nacional del Fenómeno El Niño (ENFEN) en el Perú, señala que hasta el mes de marzo de 2016 la probabilidad que la magnitud del ENSO sea moderada es del $50 \%$, mientras que, el escenario fuerte tendría una probabilidad de $35 \%{ }^{\left({ }^{8}\right)}$. Ante ello, se vienen desarrollando múltiples esfuerzos sectoriales e institucionales que permitirán identificar las áreas con mayor vulnerabilidad ante la presencia del ENSO y así transferir recursos para actividades de mantenimiento, acondicionamiento y recuperación. Por lo tanto, la identificación y el conocimiento de los potenciales riesgos a la infraestructura y funcionamiento del sector Salud proporcionarán información valiosa para la formulación de estrategias y acciones orientadas a minimizar los posibles daños en este sector.

Los episodios de ENSO generan cuantiosas pérdidas, el Ministerio de Economía y Finanzas (MEF) reportó en 1998 pérdidas por USD 3,5 mil millones $(6,2 \%$ del PBI), similar a lo ocurrido en el 1982-1983 que ocasionó daños por USD 3,3 mil millones (11,6\% del $\mathrm{PBI})^{(9)}$, con un mayor impacto en los departamentos de Tumbes, Piura, Lambayeque y La Libertad; asimismo, se destinaron aproximadamente USD 4,8 millones para la compra de medicinas, material médico, movilización de brigadas y atención a la población afectada. Del total de EE. SS. afectados en el último fenómeno, el $60 \%$ estuvo localizado en la costa norte, la mayoría fueron puestos de salud, siendo el departamento de Piura el más afectado ( $21,5 \%$ del total de establecimientos). Además, durante el evento del año 1997-1998, los gastos realizados en la rehabilitación y reconstrucción de un hospital y cinco centros de salud representaron un aproximado de USD 34 millones ${ }^{(10)}$.

Los sistemas de información geográfica (SIG) son herramientas útiles para visualizar, analizar e interpretar datos de localización geográfica durante el curso de un desastre natural y/o el planeamiento preventivo (11). Debido a ello, el objetivo del presente estudio fue estudiar la utilidad de una simulación espacial para estimar la posible vulnerabilidad de los EE. SS. públicos en el norte del Perú ante los posibles efectos del ENSO.

\section{MATERIALES Y MÉTODOS}

\section{DISEÑO DEL ESTUDIO}

Se realizó un análisis espacial exploratorio tomando como área de estudio a cuatro regiones costeras del norte del Perú que fueron seriamente afectadas por el ENSO de 1997-1998. El resultado del análisis se presenta mediante la construcción de mapas temáticos que proyectan áreas de influencia desde los puntos de riesgo hacia los establecimientos de salud ubicados hasta los $1500 \mathrm{~m}$ en cada región.

\section{REGIONES DE ESTUDIO}

Las regiones incluidas en el estudio fueron: Tumbes, Piura, Lambayeque y La Libertad. La superficie de Tumbes es de $4669 \mathrm{~km}^{2}$, con una población proyectada por el Instituto Nacional de Estadística e Informática (INEI) para el 2015 de 237685 habitantes y 43 EE. SS. públicos georreferenciados según datos del Registro Nacional de Establecimientos de Salud (RENAES). Por su parte, Piura cuenta con un área de 35892 $\mathrm{km}^{2}$, con una población proyectada para el 2015 de 1 844129 habitantes y 388 EE. SS. georreferenciados según RENAES. A su vez, Lambayeque tiene una extensión de $14231 \mathrm{~km}^{2}$, con una población proyectada 
para el 2015 de 1260650 habitantes y 177 EE. SS. georreferenciados según RENAES. La Libertad tiene un área aproximada de $25569 \mathrm{~km}^{2}$, con una población proyectada para el 2015 de 1859640 habitantes y 264 EE. SS. georreferenciados.

\section{FUENTES DE INFORMACIÓN}

Para la elaboración del presente estudio se usaron cuatro fuentes de información. La primera de ellas corresponde a la Autoridad Nacional del Agua (ANA) del Ministerio de Agricultura y Riego del Perú, de donde se obtuvieron las áreas vulnerables en época de lluvias extremas en 21 regiones del Perú. El informe publicado proporciona la ubicación georreferenciada mediante coordenadas UTM (Universal Transverse Mercator) de 563 puntos vulnerables ante daños de manera directa. Para el presente trabajo se obtuvieron las coordenadas geográficas de cada uno de los puntos de riesgos para las cuatro regiones en estudio que fueron ingresados a una hoja de cálculo para su posterior procesamiento.

La segunda fuente corresponde a los datos de los EE. SS. que se obtuvieron del RENAES por intermedio de la Oficina de Estadística del Ministerio de Salud (MINSA). La relación de establecimientos de las regiones seleccionadas para el estudio contiene datos de denominación, categorización, dirección, ubicación distrital y provincial, entre otros. Para la categorización de los EE. SS. se empleó la Norma Técnica "Categorías de Establecimientos del sector Salud" establecida por el MINSA: categoría I-1, que se corresponde con un puesto de salud o posta de salud con profesional de salud no médico; categoría I-2 que corresponde a un puesto de salud con médico; la categoría I-3 incluye centros de salud sin internamiento; la categoría I-4 se corresponde con un centro de salud con camas de internamiento; la categoría II incluye hospitales de atención general y/o especializada, y la categoría III abarca hospitales e institutos especializados ${ }^{(12)}$.

La tercera fuente de información corresponde a la obtención del mapa político del Perú con límites regionales y provinciales para la proyección de los puntos y representación geográfica, los cuales fueron obtenidos del Instituto Nacional de Estadística e Informática, para fines del estudio estos mapas se dividieron por cada una de las regiones para una mejor visualización.

La cuarta fuente proporcionó las representaciones de ríos y quebradas para cada una de las regiones de estudio, estos fueron obtenidos del sitio web de The Humanitarian Data Exchange (https://data.hdx.rwlabs. org/dataset/hidrografia-de-peru).

\section{ANÁLISIS DE DATOS}

Para el procesamiento de datos y análisis espacial, se utilizó el software ArcGIS Desktop versión 10.4 (ESRI Inc., Redlands, CA, USA), mediante el cual se integraron las cuatro fuentes de información en formato shapefiles. La integración se inició con la transformación de los datos en diferentes sistemas de coordenadas a un único sistema (WGS 1984 UTM Zone 17S), para luego transformar cada punto georreferenciado a capas vectoriales mediante archivos shapefiles. Finalmente, se tuvieron como capas resultantes: una de EE. SS., una de puntos de riesgo, una de hidrología y una capa base de regiones.

Después de integrar los datos, se procedió a seleccionar cada EE. SS. para intersectarlo con áreas de influencia concéntricas desde las zonas de riesgos. Esta metodología es conocida dentro de los SIG como buffer múltiple y sirve para evaluar la dependencia espacial entre dos elementos según distancia. Para la presente simulación, al no encontrar en la literatura estudios que incorporen la variabilidad geográfica peruana, el criterio de los investigadores fue crear tres buffer con un radio o distancia de $200 \mathrm{~m}$, de 200 hasta $1000 \mathrm{~m}$ y de 1000 a $1500 \mathrm{~m}$ para expresar un mayor o menor riesgo según la menor o mayor distancia al punto de la zona de riesgo. De esta forma se crean circunferencias con radios establecidos tomando como punto central la zona de riesgo georreferenciada obtenida por la ANA, y se determinan los puntos de cada EE. SS. georreferenciado que se encuentran dentro de las áreas de afectación del buffer.

\section{CONSIDERACIONES ÉTICAS}

Los datos fueron obtenidos de dominios públicos y/o de libre acceso que no incluye información de seres humanos. En tal sentido no se requiere la aprobación de un comité de ética.

\section{RESULTADOS}

En la región Tumbes la ANA reportó 30 puntos de riesgo. En la Figura 1 se muestran los 30 puntos de riesgo con sus respectivos buffers o áreas de influencia para la región. Según el área de influencia, de un total de 43 EE. SS. que existen en la región, dos se encuentran dentro de los 200 m; once entre 200 y 1000 m, y tres entre 1000 y 1500 m de radio al punto de riesgo. De acuerdo con la categoría de los EE. SS. con posible afectación, cuatro tenían categorización I-1; tres eran I-2; cinco fueron I-3, y cuatro pertenecían a la categoría l-4.

En Piura se reportaron 39 puntos de riesgo. Según el área de influencia, de los 388 EE. SS. georreferenciados 


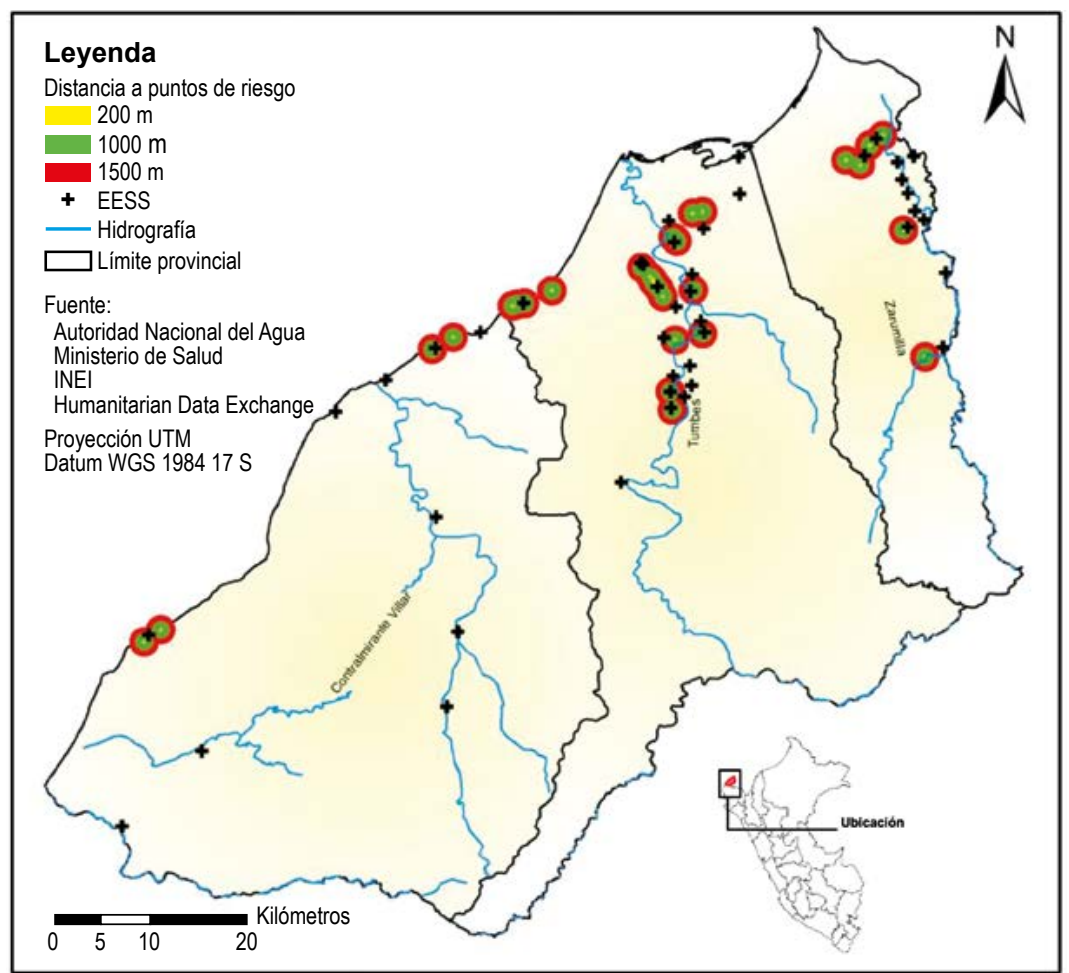

Figura 1. Áreas de influencia de puntos de riesgo y establecimientos de salud públicos en la región Tumbes.

de la región, uno se encontraba dentro de los $200 \mathrm{~m}$; nueve entre 200 y $1000 \mathrm{~m}$, y dos entre 1000 y $1500 \mathrm{~m}$ de radio del punto de riesgo identificado por la ANA. Por categorías de los EE. SS. con posible afectación, tres corresponden a la I-1; tres de categorización I-2; cinco eran I-3, y uno de la categoría I-4.

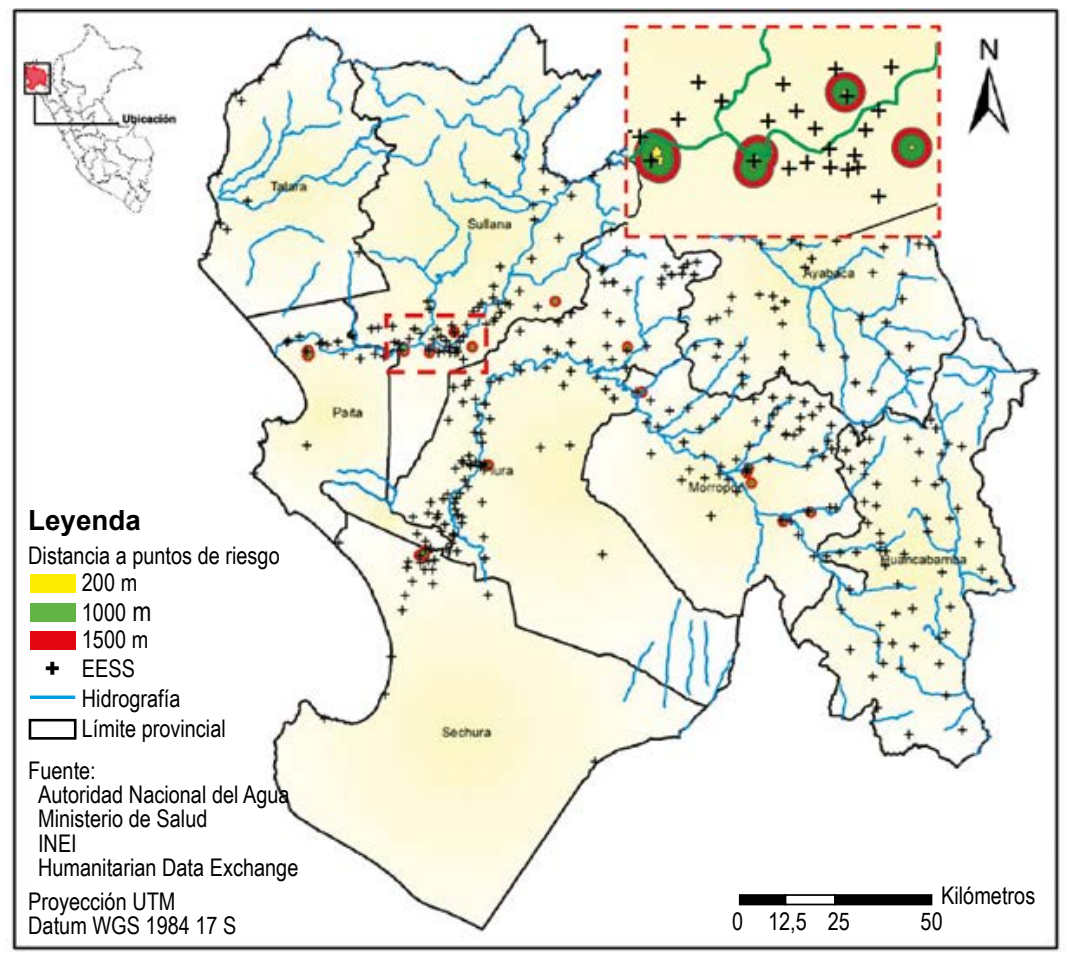

Figura 2. Áreas de influencia de puntos de riesgo y establecimientos de salud públicos en la región Piura. 
En Lambayeque se identificaron 31 puntos de riesgo. Según el área de influencia, de los 177 EE. SS. de la región, dos se encuentran entre 200 y $1000 \mathrm{~m}$, y dos entre 1000 y $1500 \mathrm{~m}$ de radio del punto de riesgo.
De acuerdo a la categoría de los EE. SS. con posible afectación, uno era I-1; dos de la categorización I-2, y uno pertenecía a la categoría l-3.

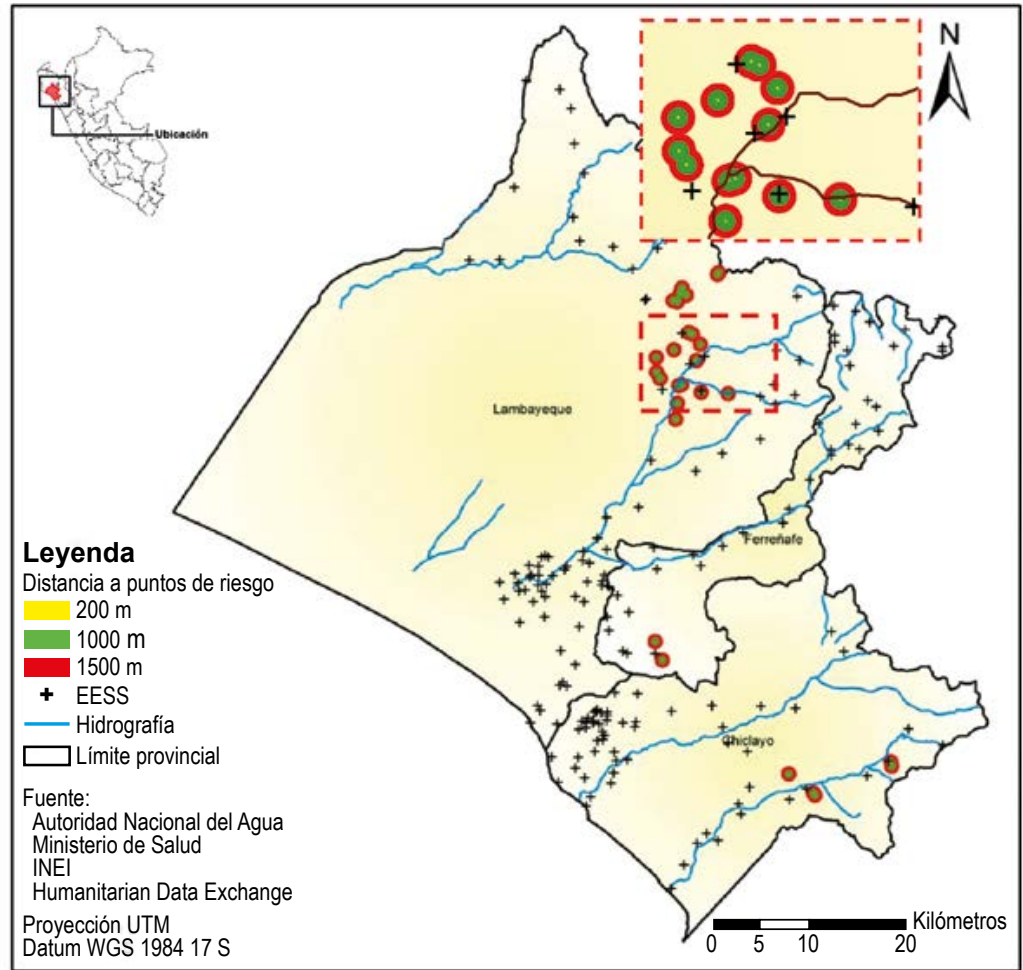

Figura 3. Áreas de influencia de puntos de riesgo y establecimientos de salud públicos en la región Lambayeque.

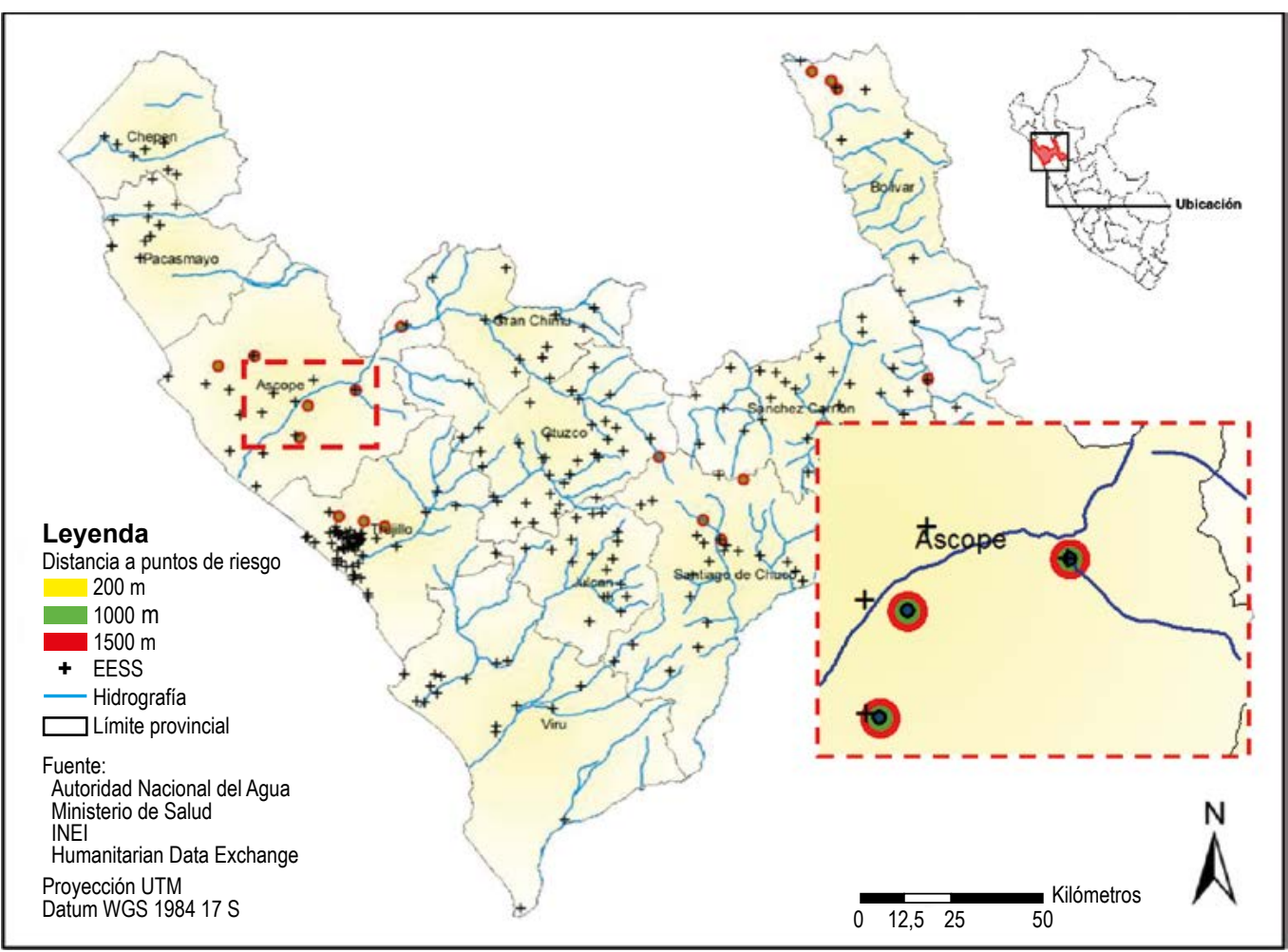

Figura 4. Áreas de influencia de puntos de riesgo y establecimientos de salud públicos en la región La Libertad. 
En La Libertad se reportaron 18 puntos de riesgo. Según el área de influencia, de los 264 EE. SS. de la región que están inscritos en RENAES, dos se encuentran dentro de los 200 m, tres entre 200 y 1000 m, y uno entre 1000 y $1500 \mathrm{~m}$ de radio del punto de riesgo. De acuerdo a la categoría de los EE. SS. con posible afectación, tres atañen a la categoría I-2; uno fue I-3, y dos comprenden a la categoría l-4.

De los 872 establecimientos estudiados, 38 (4,4\%) serían afectados ante un evento de ENSO que genere lluvias extremas. La región de Tumbes podría ser la más afectada puesto que $37,2 \%$ de sus establecimientos están cerca de los puntos de riesgo; luego se encuentra Piura con el $3,1 \%$ seguido de Lambayeque y $\mathrm{La}$ libertad con un $2,3 \%$, respectivamente. En cuanto a la categorización de los establecimientos posiblemente afectados, la categoría I-2 y I-3 serían las más afectadas con el $28,9 \%$ y $31,6 \%$ respectivamente. Según el área de influencia para los puntos de riesgo, el $60,5 \%$ de los establecimientos con posible afectación estarían entre los 200 y 1000 m (Tabla 1).

Tabla 1. Número y características de los establecimientos de salud afectados

\begin{tabular}{lcc}
\hline & $\begin{array}{c}\text { Establecimientos } \\
\text { afectados }(\mathbf{N}=38)\end{array}$ & $(\%)$ \\
\hline $\begin{array}{l}\text { Establecimientos } \\
\text { afectados por región }\end{array}$ & 16 & $(42,1)$ \\
\hline Tumbes & 12 & $(31,6)$ \\
\hline Piura & 4 & $(10,5)$ \\
\hline Lambayeque & 6 & $(15,8)$ \\
\hline La Libertad & & \\
\hline $\begin{array}{l}\text { Categoría del } \\
\text { establecimiento }\end{array}$ & 8 & $(21,1)$ \\
\hline Categoría I-1 & 11 & $(28,9)$ \\
\hline Categoría I-2 & 12 & $(31,6)$ \\
\hline Categoría I-3 & 7 & $(18,4)$ \\
\hline Categoría I-4 & & \\
\hline Área de influencia & 5 & $(13,2)$ \\
\hline Hasta $200 \mathrm{~m}$ & 25 & $(65,8)$ \\
\hline 200 a $1000 \mathrm{~m}$ & 8 & $(21,1)$ \\
\hline 1000 a1500 m & & \\
\hline
\end{tabular}

\section{DISCUSIÓN}

Se encontró una potencial vulnerabilidad de EE. SS. ubicados dentro de los $200 \mathrm{~m}$ hasta los $1500 \mathrm{~m}$ del área de influencia de los puntos de riesgo georreferenciados de presentarse un episodio de ENSO. Las áreas identificadas mediante la simulación y análisis espacial nos permiten relacionar zonas de riesgo con la localización de los establecimientos de salud públicos de las cuatro regiones estudiadas que, potencialmente, podrían ser afectados por inundaciones y deslizamientos de tierra.

Se debe tener en cuenta que el impacto de un desastre natural es el resultado de la interacción de múltiples eventos, factores y actores. Por lo tanto, estar prevenidos ante desastres naturales es una tarea dinámica $y$ compleja, en donde el Estado, las autoridades y la población desempeñan un rol clave. En ese sentido, variadas son las experiencias en donde el uso de los SIG han contribuido en la prevención de daños ante desastres naturales ${ }^{(13,14)}$, además de su posible utilidad en la elaboración de intervenciones en todas las fases de la gestión de riesgos ${ }^{(15-17)}$. El empleo de los SIG no solo aplica a la toma de decisiones preventivas ante la posible ocurrencia de desastres; el acceso a la tecnología por parte de los ciudadanos permite el uso de estas herramientas en tiempo real dando la posibilidad de entregar y obtener reportes a quien disponga de un equipo tecnológico para ello. De esta manera, se propicia una interacción personas-instituciones que, incluso, puede superar en calidad y consistencia a la información obtenida por instituciones nacionales, puesto que se crea un esfuerzo colectivo en la creación de datos georreferenciados. El desafío sectorial es crear las aplicaciones necesarias que permitan de una manera simple e intuitiva la entrega de datos a las instituciones con el objeto que estas puedan reaccionar rápida y eficazmente en canalizar la acción pública ${ }^{(18)}$.

En nuestro estudio, la mayoría de establecimientos potencialmente afectados en las cuatro regiones serían de categoría I, esto posiblemente debido a un mayor número de EE. SS. de este tipo en el Perú, su localización en zonas rurales y mayor cercanía a los puntos de riesgo señalados por la ANA. Esta afectación interrumpiría la atención de pacientes según nivel resolutivo, con una mayor demanda hacia EE. SS. de mayor complejidad (categoría II y III). Ante ese posible escenario, el MINSA y la Seguridad Social (EsSalud) han establecido planes de contingencia para atender la sobredemanda y necesidades ante una situación de emergencia ${ }^{(19,20)}$.

Debido a la probabilidad de ocurrencia del ENSO, el Ministerio de Economía y Finanzas del Perú autorizó una transferencia presupuestaria del gobierno central destinada a financiar la ejecución de actividades y proyectos de reducción de riesgos, preparación y respuesta en las zonas declaradas en estado de emergencia ante el período de lluvias 2015-2016 y la ocurrencia del ENSO ${ }^{(21)}$. Asimismo, otras instituciones como el Ministerio de Agricultura y Riego, emplean recursos para la limpieza de ríos, drenes y quebradas en las regiones declaradas en emergencia ${ }^{(22)}$. Por tanto, el 
contar con un reporte de EE. SS. previamente afectados y/o con posibilidad de afectación ante desastres naturales permitiría disponer de forma más eficiente los recursos destinados.

La mitigación de daños ante desastres naturales puede darse con medidas estructurales que incluyan códigos de construcción y especificaciones de materiales, reacondicionamiento de las estructuras existentes aumentando su resistencia a desastres y la construcción de diques $u$ otros mecanismos protectores. Si bien estas medidas a corto plazo pueden ser eficaces, tiene un costo directo asociado al costo del proyecto de mejora y, a la larga, tienden a generar mayores problemas, además de una "sensación de seguridad" no necesariamente acorde con la realidad. Por otro lado, el empleo de medidas de mitigación no estructurales, que incluyen: ordenamiento territorial, incentivos tributarios, programas de seguros y reubicación de residentes fuera del área de riesgo, persiguen identificar zonas de riesgo y limitar el uso de las mismas. Para los países en desarrollo, como el caso de Perú, son de utilidad ya que los gastos producidos por este tipo de medidas suelen ser menores que los de cambios estructurales. Por lo tanto, es válido decir que en los países en desarrollo se debe enfatizar el uso de medidas de mitigación no estructurales, las cuales pueden ser implementadas por ONG y/o el sector privado y por autoridades centrales mediante la legislación y el respeto a los códigos de edificación y a la zonificación. A pesar de esto, no deja de tener sus propios inconvenientes, por lo cual debe estudiarse el impacto de cualquiera de estas dos formas de mitigación ${ }^{(23,24)}$.

El empleo de SIG por parte de instituciones estatales, como herramienta informativa del estado actual $y$ dinámica de las problemáticas del país y su inclusión dentro de los reportes nacionales, permite delimitar territorios que presenten mayores necesidades y, de esta forma, mejorar el destino de los fondos, que escasos, deberían en lo posible destinarse al desarrollo de las zonas menos favorecidas. El empleo de una simulación muestra una manera de abordar la gestión de desastres por medio de los SIG. Estas herramientas tienen aplicabilidad a diversos campos y temas que a nivel de desastres permitirían estimar los efectos esperados de los fenómenos naturales. Sin embargo, el uso de estas herramientas requiere una diversidad de información y el trabajo intersectorial primero en la definición de los fines y objetivos que se persiguen con la generación de la información, y segundo con las actividades y obtención de la información previamente planificada.

Como limitaciones del estudio debemos señalar que el análisis se realizó empleando información secundaria de diferentes fuentes, con la posibilidad de que los puntos georreferenciados no empleen el mismo sistema de proyección; sin embargo, durante el empleo de los mismos se realizó la conversión a un único sistema con un buen ajuste para la capa final. Cabe precisar que nuestra simulación no consideró parámetros de altitud o pendiente entre la ubicación del establecimiento y punto de riesgo que implicaría un modelamiento de mayor complejidad para una unidad de análisis menor a la incluida en el estudio. Asimismo, el no disponer de una base actualizada de los EE. SS. o la falta de georreferenciación podría estar mostrando un menor número de establecimientos potencialmente afectados con el análisis empleado. Sería recomendable contar con reportes de evaluación de riesgos por parte del sector salud para los EE. SS., esta medida enriquecería la información sobre afectación de los mismos, permitiendo que las medidas de contingencia se establezcan de forma más acorde con la realidad de cada EE. SS. Por otro lado, el estudio se basa en una simulación de buffers, por lo que, idealmente, se debería integrar en las fuentes de información datos de hidrología, incluyendo históricos de caudales y/o cauces, además de incluir la topografía para enriquecer aun más el análisis y disponer de información con mayor precisión.

En conclusión, se identificaron EE. SS. cercanos a puntos de riesgo de inundaciones que deben ser evaluados en el terreno para determinar su real vulnerabilidad y así se puedan generar acciones preventivas frente a desastres naturales. Nuestro estudio muestra la utilidad de contar con sistemas de información geográfica en la gestión y planificación de la infraestructura pública en salud, teniendo en cuenta las características geográficas del territorio. No obstante, la implementación de plataformas basadas estos sistemas son de alto costo, es por ello que se hace relevante la coordinación interinstitucional en la creación de datos espaciales con estándares homologados que permitan la superposición de las capas, como también la creación de una plataforma abierta que permita la integración, visualización y descargas de los mismos. Asimismo, es de vital importancia capacitar a los decisores en el uso de estas herramientas, con el propósito de que incorporen el análisis territorial en el ejercicio de sus funciones. Para ello existen softwares libres y de código abierto que permiten el desarrollo de los sistemas de información geográfica sin la necesidad de contar con un alto presupuesto.

Contribuciones de autoría: AHV tuvo la idea de investigación. $A H V, G B Q$ y DDS diseñaron el estudio y recopilaron los datos. AHV, DDS y EG realizaron el procesamiento espacial de los datos. AHV, HAH, GBQ, DDS, SV, JRG y EG analizaron e interpretaron los datos, redactaron el manuscrito y aprobaron la versión final.

Fuentes de financiamiento: autofinanciado.

Conflictos de interés: los autores declaran no tener conflictos de interés. 


\section{REFERENCIAS BIBLIOGRÁFICAS}

1. Gonzales GF, Zevallos A, GonzalesCastaneda C, Nunez D, Gastanaga C, Cabezas C, et al. [Environmental pollution, climate variability and climate change: a review of health impacts on the Peruvian population]. Rev Peru Med Exp Salud Publica. 2014;31(3):547-56. doi: http://dx.doi.org/10.17843/ rpmesp.2014.313.94

2. Sanabria-Rojas H. Panorama epidemiológico de los desastres naturales. Ensayo a propósito del fenómeno El Niño en el Perú (1998). Rev Peru Med Exp Salud Publica. 1998;15(1-2):37-44.

3. Suarez-Ognio L, Estela-Ayamamani D, Caceres-Mejia B, Gambirazio-Carbajal C, Cabrera R. [Impact of the "El Niño" phenomenon from 1997 to 1998 on the health of the Peruvian population: risk potential for 2015]. Rev Peru Med Exp Salud Publica. 2015;32(2):4034. doi: http://dx.doi.org/10.17843/ rpmesp.2015.322.1645

4. Miranda JJ. El Niño and health. Lancet. 2004;363(9404):247-8. doi: http://dx.doi.org/10.1016/S01406736(03)15346-9.

5. Kovats RS, Bouma MJ, Hajat $S$, Worrall E, Haines A. El Niño and health. Lancet. 2003;362(9394):14819. doi:http://dx.doi.org/10.1016/ S0140-6736(03)14695-8

6. Bambaren CV, Alatrista MDS. Hospitales seguros ante desastres. Rev Med Hered. 2007;18(3):149-54. doi: http://dx.doi.org/10.20453/rmh. v18i3.923

7. Public health guide in emergencies $2^{\text {nd }}$ Ed. [Internet]. Geneva: The Johns Hopkins and the International Federation of Red Cross and Red Crescent Societies; 2008 [citado el 20 de diciembre de 2015]. Disponible en: http://www.jhsph.edu/research/ centers-and-institutes/center-forrefugee-and-disaster-response/ publications_tools/publications/_ CRDR_ICRC_Public_Health Guide_Book/Forward.pdf

8. Comité Multisectorial encargado del Estudio Nacional del Fenómeno El Niño [Internet]. Lima, Perú: Comunicado Oficial ENFEN No21-2015; 2015 [citado el 10 de diciembre de 2015]. Disponible en: http://www.senamhi.gob.pe/load/ file/02204SENA-50.pdf

9. Marco Macroeconómico Multianual 2016-2018 [Internet]. Lima: Ministerio de Economía y Finanzas; 2015 [citado el 18 de diciembre de 2015]. Disponible en: http://www. bcrp.gob.pe/docs/Publicaciones/ Programa-Economico/mmm-20162018-abril.pdf.

10. Informe Técnico 1. Impactos del fenómeno El Niño (FEN) en la economía regional de Piura, Lambayeque y la Libertad. $1^{\text {a }}$ Ed. [Internet]. Lima: Proyecto Seguros para la Adaptación al Cambio Climático; 2012 [citado el 12 de diciembre de 2015]. Disponible en: http:// seguros.riesgoycambioclimatico.org/ publicaciones/Informe-Tecnico1.pdf.

11. Kistemann $T$, Dangendorf $F$, Schweikart J. New perspectives on the use of Geographical Information Systems (GIS) in environmental health sciences. Int J Hyg Environ Health. 2002;205(3):169-81.

12. Perú, Ministerio de Salud. Resolución Ministerial 546-2011/MINSA. (16 de julio de 2011).

13. Kawasaki A, Berman ML, Guan W. The growing role of web-based geospatial technology in disaster response and support. Disasters. 2013;37(2):201-21.doi: 10.1111/j.1467-7717.2012.01302.x.

14. Kuwabara K, Matsuda S, Fushimi K, Ishikawa KB, Horiguchi H, Fujimori $\mathrm{K}$. Contribution of the administrative database and the geographical information system to disaster preparedness and regionalization. Am J Disaster Med. 2012;7(2):95-103.

15. Chen P, Zhang J, Zhang L, Sun Y. Evaluation of resident evacuations in urban rainstorm waterlogging disasters based on scenario simulation: Daoli district (Harbin, China) as an example. Int J Environ Res Public Health. 2014;11(10):9964-80. doi: 10.3390/ ijerph111009964.

16. Wilson JL, Little R, Novick L. Estimating medically fragile population in storm surge zones: a geographic information system application. J Emergen Manag. 2013;11(1):9-24.

17. Evaluación preliminar de riesgos de inundación y selección de áreas con riesgo potencial significativo en zonas costeras de la demarcación hidrográfica del Segura [Internet]. Madrid: Ministerio de Medio Ambiente y Medio Rural y Marino; 2010 [citado el 20 de diciembre de 2015]. Disponible en: https://www.chsegura. es/static/riesgoinundaciones/EPRI_ COSTAS_2-12-10.pdf.

18. Haklay ME, Antoniou V, Basiouka $S$, Soden R, Mooney P. Crowdsourced Geographic Information use in Government. Report to Global Facility for Disaster Reduction and Recovery (World Bank). London: World Bank; 2014.

19. Perú, Ministerio de Salud. Resolución Ministerial 727-2014/MINSA. (25 de septiembre de 2014).

20. Perú, EsSalud. Resolución de Gerencia General 1179-GG-ESSALUD-2015. (28 de septiembre de 2015).

21. Perú, Ministerio de Economía y Finanzas. Decreto Supremo 281-2015EF. (25 de septiembre de 2015).

22. Plan y acciones MINAGRI frente al fenómeno el Niño 2015-2016 [Internet]. Lima: Ministerio de Agricultura y Riego; 2015 [citado el 20 de diciembre de 2015]. Disponible en: http://minagri.gob.pe/portal/ download/pdf/preparate-peru/planaccion-minagri.pdf

23. Gestión de Riesgo de Desastres Naturales [Internet]. Washington, D.C.: Banco Interamericano de Desarrollo; 2008 [citado el 20 de diciembre de 2015]. Disponible en: http://www.bvsde.paho.org/bvsacd/ cd47/riesgo.pdf.

24. Desastres, Planificación y Desarrollo: Manejo de Amenazas Naturales para Reducir los Daños [Internet]. Washington, D.C.: Organización de los Estados Americanos; 1991 [citado el 20 de diciembre de 2015]. Disponible en: http://www.oas.org/dsd/publications/ unit/oea 57 s/oea 57 s.pdf.

Correspondencia: Akram Hernández Vásquez Dirección: Calle Gavilán 3856, Ciudad Autónoma de Buenos Aires, Argentina Teléfono: (0054) 91122981572

Correo electrónico:akram.hernandez.v@upch.pe 